\title{
Adsorción de Trihalometanos en solución acuosa utilizando Zeolita natural modificada
}

\author{
Adsorption of Trihalomethanes in aqueous solution using modified natural Zeolite
}

\begin{abstract}
Víctor Manuel Sánchez-Corrales ${ }^{1}$, Jesús Manuel Quiroz Castillo*², Roberto Leyva Ramos ${ }^{3}$, Octavio Cota Arriola ${ }^{2}$, Julieta Leyva Pacheco ${ }^{2}$, Daniel García Bedoya ${ }^{2}$, Roberto Ramírez Leal ${ }^{2}$, Martín Eusebio Cruz Campas ${ }^{2}$

1 Departamento de Ingeniería Química y Metalurgia. Universidad de Sonora. Luis Encinas y Rosales S/N, Colonia Centro, C.P. 83000, Hermosillo, Sonora, México.

2 Programa Educativo de Ingeniería Ambiental, Unidad Académica Hermosillo. Universidad Estatal de Sonora. Ley Federal del Trabajo e Israel González S/N, Colonia Apolo, C.P. 83100, Hermosillo, Sonora, México.

3 Facultad de Ciencias Químicas. Universidad Autónoma de San Luis Potosí. Álvaro Obregón No. 64, Colonia Centro, C.P. 78000, San Luis Potosí, San Luis Potosí, México.
\end{abstract}

\section{RESUMEN}

Los trihalometanos (THMs) se producen cuando los desinfectantes que contienen cloro reaccionan con materia orgánica natural presente en el agua a tratar, la velocidad de formación de estas sustancias disminuye con el tiempo de contacto, pero aumenta con la dosis de cloro. Durante 1989 se examinaron las concentraciones de subproductos de la desinfección en 35 plantas de tratamiento en EE.UU. y se encontró que los THMs eran los compuestos presentes en mayor cantidad, los cuatro THMs más comunes son el triclorometano (cloroformo), dibromoclorometano, diclorobromometano y tribromometano (bromoformo). Con el fin de estudiar una posible solución a este problema, en este trabajo se realizaron pruebas de adsorción con zeolita natural del Estado de Sonora, modificada superficialmente con el surfactante hexadeciltrimetilamonio (HDTMA), para determinar su capacidad de remoción de trihalometanos en solución acuosa; se desarrollaron los experimentos de adsorción a seis distintas concentraciones y a tres diferentes temperaturas, todos los datos de equilibrio se ajustan a la ecuación de Langmuir, se pueden observar ligeras diferencias en la capacidad de adsorción para cada contaminante, detectándose una mayor capacidad para dibromoclorometano y bromoformo. La fase hidrofóbica en la superficie de la zeolita se comporta como un medio de partición para los THMs, por lo que la zeolita modificada con HDTMA puede ser utilizada para adsorber THMs en solución acuosa.

Palabras clave: Trihalometanos, zeolita, adsorción.

\section{ABSTRACT}

Trihalomethanes (THMs) are produced when chlorine, used in water disinfection, reacts with natural organic matter. The rate of THMs formation decreases with contact time, but increases with chlorine dose. In 1989, the concentration of disinfection by-products were analyzed in 35 treatment plants in the U.S.A., finding that THMs were the byproducts of disinfection present in greater quantities; the four most common THMs are trichloromethane (chloroform), dibromo- chloromethane, dichlorobromomethane and tribromomethane (bromoform). In order to study a possible solution to this problem, in this research, adsorption tests were performed with natural zeolite from the State of Sonora, surface modified with hexadecyltrimethylammonium surfactant (HDT$M A)$, to determine its capacity to remove trihalomethanes in aqueous solution. We carried out adsorption experiments for trihalomethanes at six different concentrations and three different temperatures, and all equilibrium data adjusted to the Langmuir equation. Slight differences in the adsorption capacity can be observed for each pollutant; with a greater adsorption capacity detected for dibromochloromethane and bromoform. The hydrophobic phase on the surface of the zeolite behaves as a partition medium for the THMs, therefore, modified zeolite with HDTMA can be used to adsorb THMs in aqueous solution.

Keywords: Trihalomethanes, zeolite, adsorption.

\section{INTRODUCCIÓN}

Los trihalometanos (THMs) se producen cuando el cloro reacciona con materia orgánica natural presente en el agua, la formación de los THMs aumenta con la dosis de cloro. Durante 1989 se examinaron las concentraciones de contaminantes en 35 plantas de tratamiento, y se encontró que los THMs eran los subproductos de la desinfección presentes en mayor cantidad (Ioannou et al., 2016), los cuatro THMs más comunes son el cloroformo, dibromoclorometano, diclorobromometano y bromoformo. De acuerdo a la Agencia Internacional de Investigación en Cáncer (IARC, por sus siglas en inglés) los THMs son agentes cancerígenos del grupo 2B (IARC, 1999); el cloroformo es el THM más común en los sistemas de distribución que usan agua superficial, no se cumple esto en sistemas que tratan agua subterránea, en la que el bromoformo es el más abundante, el dibromoclorometano es el que produce un mayor riesgo a padecer cáncer, seguido por el bromoformo, cloroformo y diclorobromometano (Font-Ribera et al., 2018). Se han realizado estudios que sugieren una conexión entre los THMs y cáncer de vejiga, 
y posiblemente cáncer de colon y recto (Min y Min, 2016), otros estudios han asociado a los THMs con problemas reproductivos, incluyendo el aumento al riesgo de abortos, tal es el caso de un estudio en California (EE.UU.) donde se observó un promedio de aborto de 15.7 \% para mujeres que bebían diariamente 5 vasos de agua fría con una concentración de $75 \mu \mathrm{g} / \mathrm{L}$ de THMs, comparado con un $9.5 \%$ presentado en mujeres expuestas a bajas concentraciones de estas sustancias (Siddique et al., 2015).

Según investigaciones, algunas maneras de reducir los niveles de THMs incluyen la remoción de materia orgánica antes de desinfectar, reducir la dosis de cloro o utilizar desinfectantes alternativos como cloroaminas, ozono y rayos UV (Zainudin et al., 2017), también se ha estudiado la remoción de THMs mediante adsorción en carbón activado granular y fibras de carbón activado, este método ha sido reconocido como un medio efectivo de purificación de agua, sin embargo, el diseño y operación de columnas empacadas con carbón activado se ha visto complicada por la presencia de materia orgánica disuelta, que es adsorbida preferencialmente en la superficie del carbón activado, evitando la adsorción de los THMs (He et al., 2017).

Las zeolitas naturales son aluminosilicatos hidratados cristalinos que poseen una estructura microporosa o andamiaje molecular, formado a base de tetraedros de $\mathrm{SiO}_{4}^{-4} \mathrm{y}$ $\mathrm{AlO}_{4}^{-5}$, unidos a través de átomos de oxígeno (Wibowo et al., 2017). El andamiaje contiene canales y cavidades con dimensiones de 3 a 10 Å ocupadas por cationes metálicos alcalinos y alcalinoterreos y moléculas de agua. Estos cationes tienen una gran libertad de movimiento y pueden ser intercambiados (Millar et al., 2016). La clinoptilolita es la zeolita natural más usada por su capacidad de adsorción e intercambio iónico y la abundancia de yacimientos (Dziedzicka et al., 2016). Las zeolitas naturales modificadas con surfactante han sido utilizadas como barreras impermeables para contaminantes en suelos (De Genaro et al., 2014), se ha utilizado clinoptilolita, modificada superficialmente con surfactantes catiónicos, con el propósito de eliminar distintos tipos de contaminantes orgánicos en agua (Wang et al., 2011), el surfactante más usado para este fin es el hexadecilmetiltrimetilamonio (HDTMA), distintos estudios sugieren que las zeolitas modificadas con HDTMA presentan una buena capacidad para adsorber compuestos como benceno, tolueno, etilbenceno y xileno (Vidal et al., 2012; Reeve y Fallowfield, 2018; Tran et al., 2018).

Con el fin de estudiar una posible alternativa a los métodos tradicionales de eliminación de THMs, en este trabajo se realizaron pruebas de adsorción con clinoptilolita natural del Estado de Sonora, modificada superficialmente con HDTMA.

\section{MATERIALES Y MÉTODOS}

\section{Materiales}

Mezcla de calibración de THMs de 2000 mg/L (Supelco), hexano grado HPLC, 1,2-dibromopropano grado HPLC, bromuro de hexadeciltrimetilamonio (HDTMA), y alcohol metílico al $99.93 \%$ (Aldrich), acetona al $99.9 \%$ y nitrato de sodio (Fermont), y clinoptilolita natural del municipio de Rayón en el Estado de Sonora. Se preparó en el laboratorio agua libre de THMs hirviendo por 60 min agua previamente destilada y desionizada, para después hacerla pasar por un lecho de carbón activado.

\section{Modificación superficial de la zeolita}

La clinoptilolita se recibió en forma de rocas grandes, éstas se redujeron de tamaño con un cincel y se molieron en un mortero de cerámica, se tamizó utilizando una malla 400, el material que pasó el tamiz se utilizó en los experimentos posteriores. Se modificó la superficie de la clinoptilolita de acuerdo al procedimiento descrito por Chao y Chen (2012) con el objetivo de que su superficie presentará una monocapa del surfactante, favoreciendo la adsorción de compuestos orgánicos volátiles; con fines comparativos, también se preparó clinoptilolita modificada presentando una bicapa de surfactante.

\section{Caracterización \\ Difracción de rayos-X}

Los patrones de difracción de rayos- $X$ de la clinoptilolita en polvo fueron obtenidos en un difractómetro Rigaku Geigerflex, usando radiación de Cu Ka $(\lambda=1.542 \AA$ A y un monocromador de grafito.

\section{Propiedades de textura}

Las propiedades de textura (área específica, diámetro promedio de los poros y volumen de poros) de la zeolita se determinaron utilizando la técnica de fisisorción de $\mathrm{N}_{2}$. Esta técnica está basada en la fisisorción de nitrógeno sobre la superficie de un sólido a temperatura de ebullición del nitrógeno líquido $(77 \mathrm{~K})$. Los datos de adsorción y desorción de $\mathrm{N}_{2}$ sobre la superficie del material se utilizan para determinar el área específica y el volumen de mesoporos. Estas propiedades de textura se calculan con la ecuación teórica de Brunauer, Emmet y Teller, BET (Buttersack et al., 2016). El análisis se realizó utilizando un equipo de fisisorción de $\mathrm{N}_{2^{\prime}}$ marca Micromeritics, modelo ASAP 2010.

\section{Potencial Z}

Las mediciones de potencial zeta se realizaron con una unidad Zeta-Meter 3.0+ (Zeta-Meter Inc.). Las muestras se prepararon con $10 \mathrm{mg}$ de clinoptilolita en $100 \mathrm{~mL}$ de solución de nitrato de sodio. Después se agitaron magnéticamente, se ajustó el pH y se acondicionaron por $15 \mathrm{~min}$. Una vez transcurrido el tiempo de acondicionamiento, se midió el pH final. Finalmente se transfirieron a la celda del medidor de potencial zeta. La medición del tiempo requerido por una partícula para recorrer una distancia conocida a través de la celda en un campo eléctrico fijo permite calcular la movilidad electroforética de la partícula. La movilidad electroforética se convierte a potencial zeta utilizando la ecuación de Helmholtz-Smoluchowski (Pollastria et al., 2014). Esta conversión la realiza automáticamente el sistema Zeta-Meter. 
Los valores reportados de potencial zeta corresponden al promedio de las lecturas de 20 partículas.

\section{Análisis termogravimétrico}

El análisis se realizó con un Analizador Termogravimétrico (TGA), marca Perkin Elmer, modelo Pyris Diamond TGA/DTA. Se pesó una muestra de $4 \mathrm{mg}$ de clinoptilolita modificada y se colocó en una cápsula de alúmina y ésta a su vez en la balanza que se encuentra dentro de la cámara del TGA. La muestra se sometió a una rampa de temperatura que consistió en calentar a $50^{\circ} \mathrm{C}$ por un minuto, posteriormente se calentó hasta $700{ }^{\circ} \mathrm{C}$ con una velocidad de calentamiento de $10^{\circ} \mathrm{C} / \mathrm{min} \mathrm{y}$, por último, se disminuyó la temperatura de $700{ }^{\circ} \mathrm{C}$ a $50^{\circ} \mathrm{C}$ utilizando un flujo de aire a una velocidad de enfriamiento de $10^{\circ} \mathrm{C} / \mathrm{min}$.

\section{Adsorción de trihalometanos}

Los datos experimentales del equilibrio de adsorción de los THMs sobre clinoptilolita modificada con surfactante se obtuvieron utilizando un arreglo experimental de laboratorio que consistió en un adsorbedor de lote. Cada sistema de adsorción consistió en un frasco de vidrio ámbar de $40 \mathrm{~mL}$, al cual se añadió una cantidad de adsorbato y adsorbente de acuerdo a las concentraciones indicadas en el diseño de experimentos para la adsorción de trihalometanos (Tabla 1). El adsorbedor se colocó en agitación a temperatura constante en una incubadora Max Q 4000. Por último, se determinó la concentración final de THMs en la solución acuosa, la cantidad de THMs adsorbida fue calculada través de un balance de masa.

\section{Determinación de concentración de trihalometanos en solución acuosa}

El método usado fue el 6232B del Standard Methods for the Examination of Water and Wastewater (Clesceri et al., 1999) que consiste en una extracción liquido-líquido con hexano, que después se inyectó en un cromatógrafo de gases Varian CP-3800 con un detector de captura de electrones y una columna DB-5 (5 \% difenil, dimetil-siloxano).

El programa de temperatura para la columna fue $40^{\circ} \mathrm{C}$ por $4.5 \mathrm{~min}$, posteriormente se incrementa la temperatura a $150{ }^{\circ} \mathrm{C}$ a una velocidad de $25^{\circ} \mathrm{C} / \mathrm{min}$, resultando un tiempo de análisis total de 9.1 minutos. Se utilizó 1-2 dibromopropano como estándar interno.

\section{RESULTADOS Y DISCUSIÓN}

La Figura 1, muestra el difractograma de la clinoptilolita natural (Figura 1), la mayoría de los picos que presenta son típicos en grados e intensidad a los de la clinoptilolita y la heulandita reportados en la literatura (Amereh et al., 2018), dos zeolitas naturales presentes en los yacimientos sonorenses, con este análisis se confirma la presencia de clinoptilolita en la muestra utilizada para los experimentos; también se detecta la presencia de cuarzo en esta muestra.

En la tabla 2 se presentan los resultados de los análisis de propiedades de textura de la clinoptilolita natural sin modificación y de la clinoptilolita modificada, ya sea presentando una configuración de HDTMA en monocapa o en bicapa en la superficie de la zeolita.

Se observa una considerable disminución del área superficial al ser modificada la superficie de la clinoptilolita, esto

Tabla 1. Diseño de experimentos para la adsorción de trihalometanos. Table 1. Experimental design for trihalomethanes adsorption.

\begin{tabular}{|c|c|c|c|c|c|}
\hline Tratamiento & $\begin{array}{l}\text { Volumen } \\
\text { total }(\mathrm{mL})\end{array}$ & $\begin{array}{l}\text { Masa de zeolita } \\
\text { natural modificada } \\
\text { (g) }\end{array}$ & $\begin{array}{l}\text { Concentración de } \\
\text { cada THM }(\mu \mathrm{g} / \mathrm{L})\end{array}$ & $\begin{array}{c}\text { Temperatura } \\
\left({ }^{\circ} \mathrm{C}\right)\end{array}$ & Réplicas \\
\hline 1 & 30 & 1 & 100 & 18 & 3 \\
\hline 2 & 30 & 1 & 200 & 18 & 3 \\
\hline 3 & 30 & 1 & 400 & 18 & 3 \\
\hline 4 & 30 & 1 & 600 & 18 & 3 \\
\hline 5 & 30 & 1 & 800 & 18 & 3 \\
\hline 6 & 30 & 1 & 1000 & 18 & 3 \\
\hline 7 & 30 & 1 & 100 & 28 & 3 \\
\hline 8 & 30 & 1 & 200 & 28 & 3 \\
\hline 9 & 30 & 1 & 400 & 28 & 3 \\
\hline 10 & 30 & 1 & 600 & 28 & 3 \\
\hline 11 & 30 & 1 & 800 & 28 & 3 \\
\hline 12 & 30 & 1 & 1000 & 28 & 3 \\
\hline 13 & 30 & 1 & 100 & 37 & 3 \\
\hline 14 & 30 & 1 & 200 & 37 & 3 \\
\hline 15 & 30 & 1 & 400 & 37 & 3 \\
\hline 16 & 30 & 1 & 600 & 37 & 3 \\
\hline 17 & 30 & 1 & 800 & 37 & 3 \\
\hline 18 & 30 & 1 & 1000 & 37 & 3 \\
\hline
\end{tabular}




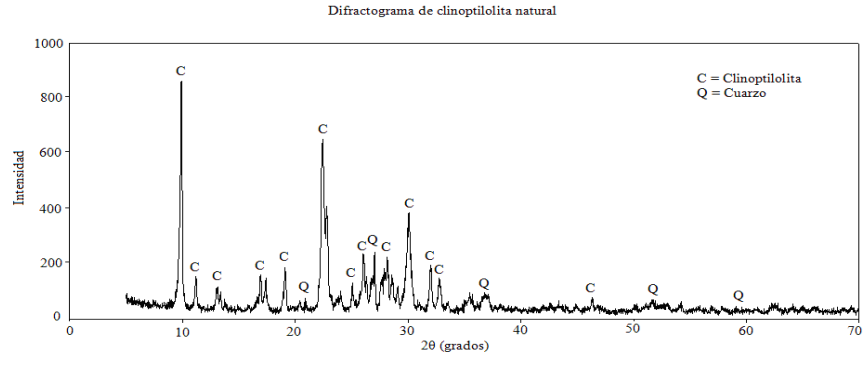

Figura 1. Difractograma de clinoptilolita natural. Figure 1. X-ray diffraction pattern of natural clinoptilolite.

Tabla 2. Propiedades de textura de la clinoptilolita natural y modificada. Table 2. Texture properties of natural and modified clinoptilolite.

\begin{tabular}{|c|c|c|c|}
\hline Muestra & $\begin{array}{l}\text { Area superficial } \\
\text { BET, } \mathrm{m}^{2} / \mathrm{g}\end{array}$ & $\begin{array}{l}\text { Porosidad } \\
\text { total, } \mathrm{cm}^{3} / \mathrm{g}\end{array}$ & $\begin{array}{c}\text { Diámetro } \\
\text { promedio de } \\
\text { poros, } \AA\end{array}$ \\
\hline Clinoptilolita & $21.7688 \pm 0.0392$ & 0.075094 & 137.9839 \\
\hline $\begin{array}{l}\text { Clinoptilolita } \\
\text { Monocapa }\end{array}$ & $8.9511 \pm 0.0141$ & 0.035135 & 157.0073 \\
\hline $\begin{array}{l}\text { Clinoptilolita } \\
\text { Bicapa }\end{array}$ & $6.8160 \pm 0.0173$ & 0.029842 & 175.1273 \\
\hline
\end{tabular}

ocurre ya que las moléculas del surfactante son demasiado grandes para penetrar en todos los poros de la clinoptilolita y se limitan a ocupar la superficie, cuando se tiene una configuración de monocapa en la superficie su área disminuye 2.4 veces y su porosidad total disminuye 2.14 veces, así mismo, el diámetro promedio de poros aumenta 1.14 veces. Cuando la configuración presente es la de bicapa, su área superficial disminuye 3.2 veces, su porosidad total disminuye 2.6 veces y el diámetro promedio de poros aumenta 1.28 veces con respecto a la clinoptilolita sin modificación.

El potencial zeta (PZ) es un reflejo del potencial de superficie. Se observó un cambio en el PZ de la clinoptilolita al ser modificada con distintas concentraciones de surfactante, se puede observar el cambio cuando se presenta monocapa y bicapa de HDTMA. La superficie progresivamente pierde su carga negativa al ponerla en contacto con el surfactante y se hace más hidrofóbica, las moléculas de surfactante son demasiado grandes como para poder penetrar los canales de la zeolita y solo tienen acceso al área externa. Al punto en que se presenta la configuración de monocapa los cationes de la superficie de la zeolita han sido solo reemplazados por HDTMA y las cadenas alquilo de éste mantienen a los aniones que interactuaban con la zeolita en solución, por lo tanto, la superficie de la zeolita modificada no presenta carga y está en su punto de mayor hidrofobicidad (Wang y Nguyen, 2015). Cuando se agrega una cantidad adicional al surfactante requerido para formar una monocapa, aparece una segunda capa de surfactante que produce un revés en la carga y la superficie vuelve a ser hidrofílica, esta presenta un máximo cuando se alcanza a formar una bicapa.

Para elucidar el efecto del $\mathrm{pH}$, se hicieron pruebas a distintos valores de éste para la clinoptilolita natural y modifi- cada, los resultados se presentan en la Figura 2. De esta figura se puede deducir que el punto en el que la carga superficial de la zeolita está más cercana al punto isoeléctrico y presenta mayor hidrofobicidad es cuando se presenta una monocapa y el sistema está a un valor de $\mathrm{pH}$ entre 6 y 7 . Variaciones en el $\mathrm{pH}$ solo alejan la carga superficial de lo que se quiere lograr para adsorber contaminantes orgánicos neutros, como lo son los THMs. Cuando se tiene una bicapa se observa que a este mismo $\mathrm{pH}$ el valor del $\mathrm{PZ}$ es alrededor de +20 , dándole hidrofilidad a la superficie y haciéndola menos adecuada para la remoción de contaminantes neutros, pero con capacidad para remover aniones en solución acuosa (Naghash y Nezamzadeh-Ejhieh, 2015).

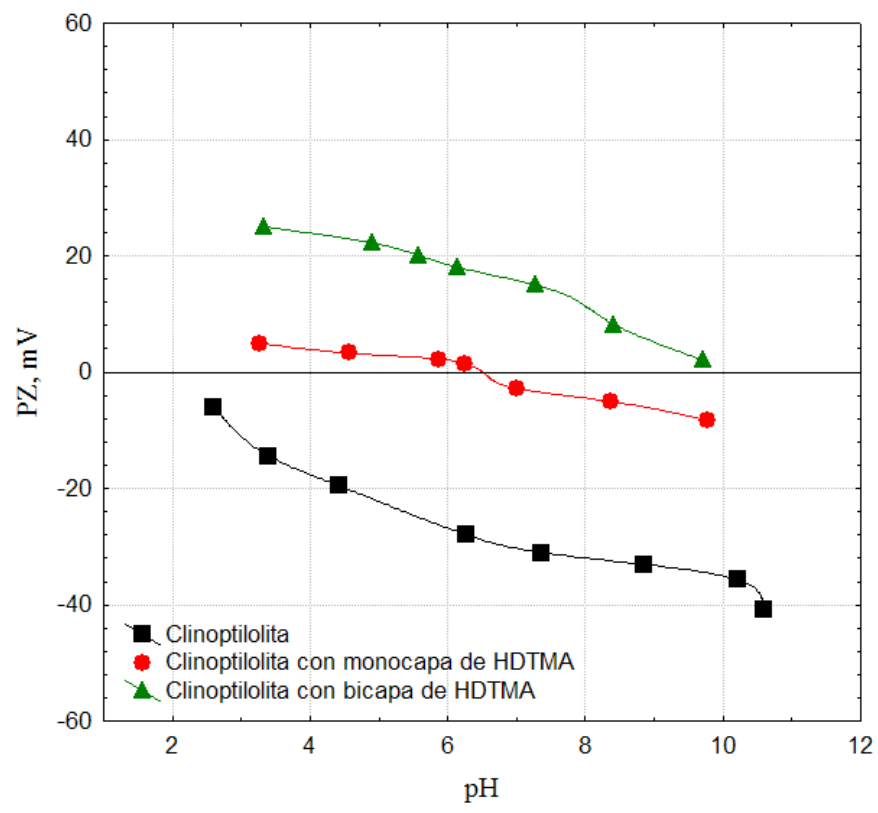

Figura 2. Efecto del pH en el potencial Zeta de la clinoptilolita natural y modificada.

Figure 2. pH Effect on Z potential of the natural and modified clinoptilolite.

En la Figura 3 se observan los resultados del análisis termogravimétrico de la clinoptilolita natural, en esta gráfica se observa la pérdida de peso y su derivada en función de la temperatura, la primera pérdida de peso que se observa es la temperatura cuando la muestra pierde el agua de hidratación y el pico que aparece después de los $300^{\circ} \mathrm{C}$ representa la pérdida del agua que estaba dentro de la celda de la zeolita, la llamada agua zeolitica (Cerri et al., 2018).

En la Figura 4 se observan los resultados del análisis termogravimétrico de la clinoptilolita cuando la configuración del surfactante en la superficie es monocapa, la característica principal de esta zeolita es su alta hidrofobicidad, y esto se corrobora al observar el pico correspondiente al agua de hidratación, que es de menor intensidad que el de la clinoptilolita natural. El segundo pico que se observa, que empieza alrededor de los $230^{\circ} \mathrm{C}$ corresponde a la fusión, evaporación y pirolisis del surfactante, el pico correspondiente a la pérdida del agua que se encuentra dentro de la celda se observa a los $400^{\circ} \mathrm{C}$. 


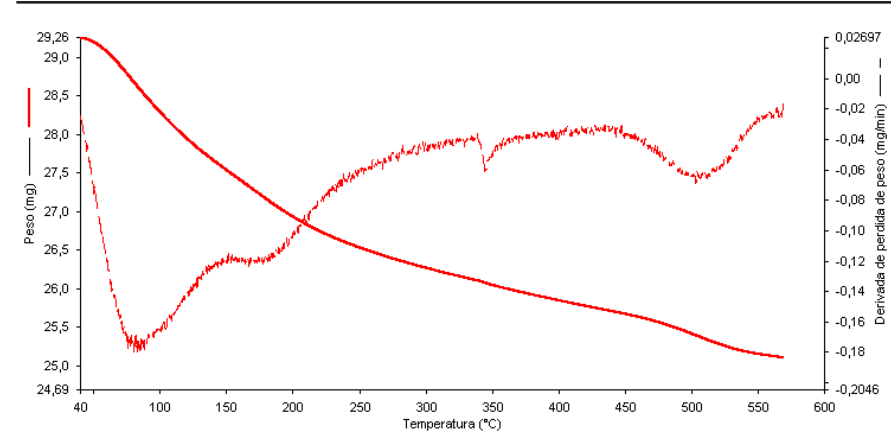

Figura 3. Termograma de clinoptilolita natural.

Figure 3. Natural clinoptilolite thermogram.

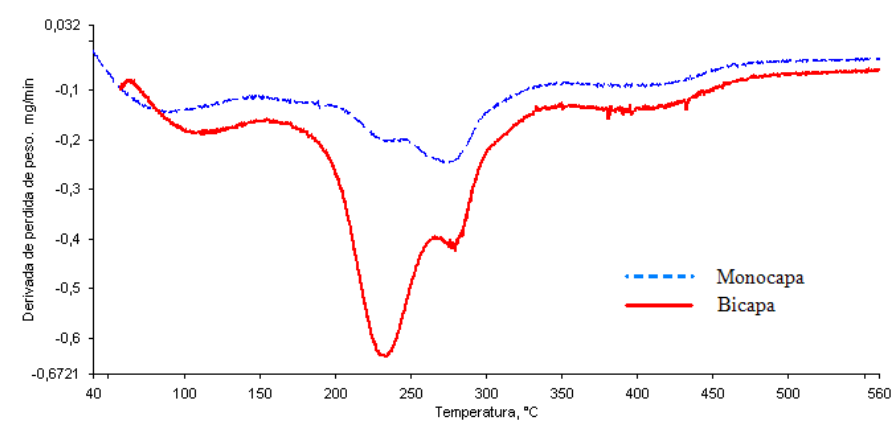

Figura 4. Comparación de termogramas de clinoptilolita modificada.

Figure 4. Modified clinoptilolite thermogram comparison.

En esta misma figura se presenta el termograma de la clinoptilolita con HDTMA en bicapa y se compara con el termograma de la monocapa de HDTMA. Se puede observar que el agua de hidratación, así como el agua de la celda, es ligeramente mayor en este caso, el pico que corresponde a la pérdida del surfactante es mucho mayor en el caso de bicapa que en el de monocapa, ya que la cantidad de HDTMA adsorbida por la zeolita en este caso es mayor.

Se seleccionó la clinoptilolita modificada con surfactante cuando éste presenta una configuración de monocapa para los experimentos posteriores, donde se evaluó la capacidad de adsorción en función de la concentración inicial de THMs y la temperatura. Todos los experimentos fueron realizados con agua libre de THMs.

La Figura 5 muestra los valores de adsorción de THMs en zeolita natural modificada a una temperatura de $28{ }^{\circ} \mathrm{C}$, estos resultados fueron obtenidos utilizando las siguientes concentraciones iniciales de THMs: 100, 200, 400, 600, 800 y $1000 \mu \mathrm{g} / \mathrm{L}$. Para todos los THMs a las distintas concentraciones iniciales se observó adsorción, adicionalmente, solo se detectaron diferencias significativas en la capacidad de adsorción de los distintos THMs cuando la concentración inicial de estos fue de $1000 \mu \mathrm{g} / \mathrm{L}$, en donde se tiene una mayor capacidad de adsorción para bromoformo y clorodibromometano, seguidos con una menor capacidad para adsorber diclorobromometano y cloroformo.Se realizaron experimentos a 18,28 y $37^{\circ} \mathrm{C}$, el THM adsorbido en mayor cantidad fue el bromoformo a una temperatura de $37^{\circ} \mathrm{C}$, alcanzando una adsorción máxima de 0.0072 mg por gramo de zeolita

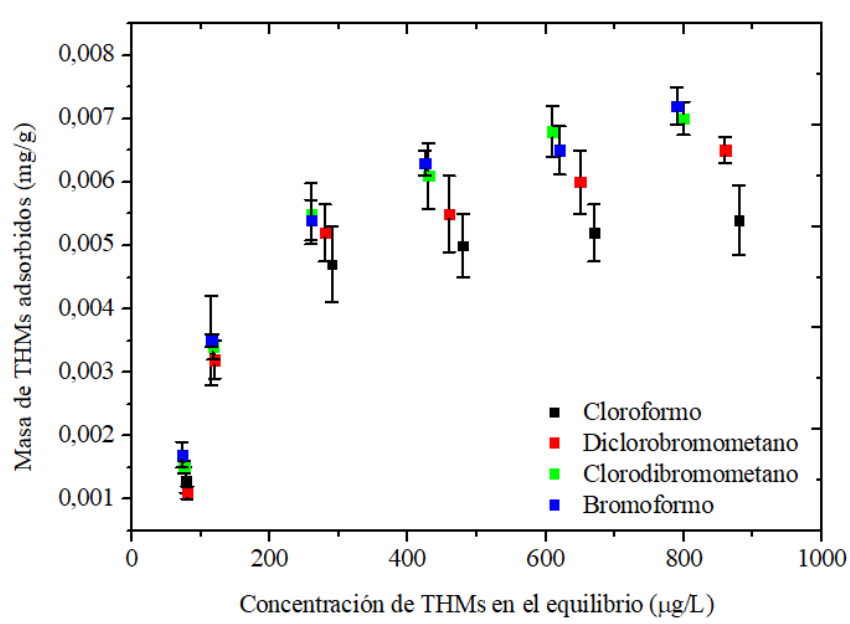

Figura 5. Adsorción de THMs en clinoptilolita natural modificada con HDTMA.

Figure 5. THMs adsorption in HDTMA modified natural clinoptilolite.

modificada, cuando la concentración inicial es de $1000 \mu \mathrm{g} / \mathrm{L}$, estos resultados se presentan en la Figura 6, también se puede observar que a partir de una concentración inicial de bromoformo de $200 \mu \mathrm{g} / \mathrm{L}$ se presenta una mayor adsorción al aumentar la temperatura del sistema, esto puede deberse a la naturaleza volátil de los contaminantes, donde el aumento de la temperatura favorece su migración a la fase sólida (He et al., 2017).

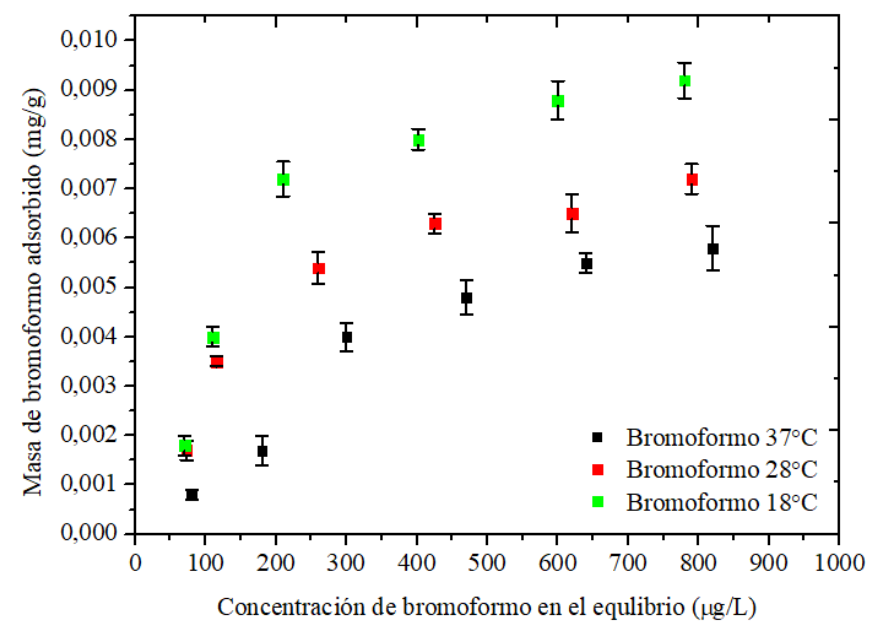

Figura 6. Efecto de la temperatura sobre la adsorción de bromoformo en clinoptilolita natural modificada con HDTMA.

Figure 6. Temperature effect on the bromoform adsorption in HDTMA modified natural clinoptilolite.

Como ha sido puntualizado por varios autores, observar un segmento lineal al inicio de la isoterma de adsorción es indicativo de un mecanismo de partición (Zainudin et al., 2017), como se ha mencionado anteriormente, la superficie hidrofílica de la zeolita puede ser modificada adsorbiendo HDTMA, formando una pseudofase orgánica o fase hidrofóbica en la superficie de la zeolita que se comporta como un medio de partición para los THMs en solución acuosa, las interacciones hidrofóbicas entre los THMs y las moléculas de 
HDTMA están controladas en su mayor parte por fuerzas de van der Walls (He et al., 2017), y consecuentemente los THMs son removidos por la zeolita modificada.

\section{CONCLUSIONES}

Se desarrolló una modificación de la zeolita natural utilizando el surfactante HDTMA, donde se logró formar una configuración de monocapa en su superficie, los cationes presentes inicialmente en la zeolita fueron reemplazados por cadenas alquilo, manteniendo a los aniones que interactuaban con la zeolita en solución, por lo tanto, la superficie de la zeolita modificada no presenta carga y está en su punto de mayor hidrofobicidad, una vez modificada, dicha superficie presenta afinidad por los THMs en solución acuosa, detectándose cierto nivel de adsorción en todas las concentraciones estudiadas, adicionalmente, la capacidad máxima de adsorción se ve favorecida al aumentar la temperatura del sistema.

La fase hidrofóbica en la superficie de la zeolita se comporta como un medio de partición para los THMs, por lo que la zeolita modificada con HDTMA puede ser utilizada para adsorber THMs en solución acuosa.

\section{REFERENCIAS}

Amereh, M., Haghighi, M. y Estifaee, P. 2018. The potential use of $\mathrm{HNO}$-treated clinoptilolite in the preparation of $\mathrm{Pt} /$ $\mathrm{CeO}_{2}$-Clinoptilolite nanostructured catalyst used in toluene abatement from waste gas stream at low temperature. Arabian Journal of Chemistry. 11: 81-90.

Buttersack, C., Mollmer, J., Hofmann, J. y Glaser, R. 2016. Determination of micropore volume and external surface of zeolites. Microporous and Mesoporous Materials. 236: 63-70.

Cerri, G., Sale, E. y Brundu, A. 2018. Thermal transformations of (NH4, Cs)- clinoptilolite with compositions in between the end-members. Microporous and Mesoporous Materials. 258: 122-130.

Chao, H.P. y Chen, S.H. 2012. Adsorption characteristics of both cationic and oxyanionic metal ions on hexadecyltrimethylammonium bromide-modified $\mathrm{NaY}$ zeolite. Chemical Engineering Journal. 193: 283-289.

Clesceri, L., Greenberg, A. y Eaton, A. 1999. Standard Methods for the Examination of Water and Wastewater. 20th ed. American Public Health Association. New York, USA.

De Gennaro, B., Catalanotti, L., Bowman, R.S. y Mercurio, M. 2014. Anion exchange selectivity of surfactant modified clinoptilolite-rich tuff for environmental remediation. Journal of Colloid and Interface Science. 430: 178-183.

Dziedzicka, A., Sulikowski, B., Ruggiero, M. y Jerzy, M. 2016. Catalytic and physicochemical properties of modified natural clinoptilolite. Catalysis Today. 259: 150-58.

Font-Ribera, L., Gràcia-Lavedana, E., Aragonés, N., PérezGómezd, B., Pollánd, M., Amianod, P., Jiménez-Zabala, A., Castaño-Vinyals, A., Roca-Barceló, A., Ardanaz, A., Burguid, R., Molina, A.J., Fernández-Villaj, T., Gómez-Acebo, I., Dierssen-Sotos, T., Moreno, V., Fernandez-Tardon, G., Peiró, R., Kogevinas, M. y Villanueva, C.M. 2018. Long-term exposure to trihalomethanes in drinking water and breast cancer in the Spanish multicase-control study on cancer (MCC-SPAIN). Environment International. 112: 227-234.
He, X., Elkouz, M., Inyanga, M., Dickenson, E. y Werta, E.C. 2017. Ozone regeneration of granular activated carbon for trihalomethane control. Journal of Hazardous Materials. 326: 101-109.

IARC. 1999. Some Chemicals That Cause Tumors of the Kidney or Urinary Bladder in Rodents and Some Other Substances. IARC Monographs on the Evaluation of Carcinogenic Risk of Chemicals to Humans, vol. 73. Lyon, France: International Agency for Research on Cancer.

loannou, P., Charisiadis, P., Syam, S., Andra S.S. y Makris, K.C. 2016. Occurrence and variability of iodinated trihalomethanes concentrations within two drinking-water distribution networks. Science of the Total Environment. 543: 505-513.

Millar, G.J., Winnett, A., Thompson, T. y Couperthwaite, S.J. 2016. Equilibrium studies of ammonium exchange with Australian natural zeolites. Journal of Water Process Engineering. 9: 4757.Min, J.Y. y Min, K.B. 2016. Blood trihalomethane levels and the risk of total cancer mortality in US adults. Environmental Pollution. 212: 90-96.

Naghash, A. y Nezamzadeh-Ejhieh, A. 2015. Comparison of the efficiency of modified clinoptilolite with HDTMA and HDP surfactants for the removal of phosphate in aqueous solutions. Journal of Industrial and Engineering Chemistry. 31: 185-191.Pollastria, S., Gualtieria, A.F., Lassinantti, M., Gualtieri, M., Hanuskova, M., Cavallo, A. y Gaudino, G. 2014. The zeta potential of mineral fibres. Journal of Hazardous Materials. 276: 469-479.Reeve, P.J. y Fallowfield, H.J. 2018. Natural and surfactant modified zeolites: A review of their applications for water remediation with a focus on surfactant desorption and toxicity towards microorganisms. Journal of Environmental Management. 205: 253-261.

Siddique, A., Saied, S., Mumtaz, M., Hussain, M.M. y Khwaja, H.A. 2015. Multipathways human health risk assessment of trihalomethane exposure through drinking water. Ecotoxicology and Environmental Safety.116: 129-136.

Tran, H.N., Viet, P.V. y Chao, H.P. 2018. Surfactant modified zeolite as amphiphilic and dual-electronic adsorbent for removal of cationic and oxyanionic metal ions and organic compounds. Ecotoxicology and Environmental Safety. 147: 55-63.

Vidal, C.B., Raulino, G.S.C., Barros, A.L., Lima, A.C.A., Ribeiro, J.P., Pires, M.J.R. y Nascimento, R.F. 2012. BTEX removal from aqueous solutions by HDTMA-modified $Y$ zeolite. Journal of Environmental Management. 112: 178-185.

Wang, H.Y., Huang, H.F. y Jiang, J.Q. 2011. The effect of metal cations on phenol adsorption by hexadecyl-trimethylammonium bromide (hdtma) modified clinoptilolite (Ct.). Separation and Purification Technology. 80: 658-662.

Wang, X. y Nguyen, A.V. 2015. Characterisation of electrokinetic properties of clinoptilolite before and after activation by sulphuric acid for treating CSG water. Microporous and Mesoporous Materials. 220: 175-182.

Wibowo, E., Sutisna, Rokhmata, M., Murniatia, R., Khairurrijal y Abdullah, A. 2017. Utilization of natural zeolite as sorbent material for seawater desalination. Procedia Engineering. 170: 8-13.

Zainudin, F.M., Hasan, H.A. y Abdullah, S.R.S. 2017. An overview of the technology used to remove trihalomethane (THM), trihalomethane precursors, and trihalomethane formation potential (THMFP) from water and wastewater. Journal of Industrial and Engineering Chemistry. 57: 1-14. 